\title{
Modélisation stochastique et robuste de l'atténuation et de l'adaptation dans un système énergétique régional. Application à la région Midi-Pyrénées
}

\author{
Christopher Andrey ${ }^{1}$, Frédéric Babonneau ${ }^{2}$, Alain Haurie ${ }^{3}$, Maryse Labriet ${ }^{4}$ \\ 1 Consultant/chercheur en modélisation énergétique, ORDECSYS, 1224 Chêne-Bougeries, Suisse \\ 2 Consultant/chercheur en modélisation énergétique, optimisation et gestion du risque, ORDECSYS, 1224 Chêne-Bougeries, Suisse \\ ${ }^{3}$ Consultant/chercheur en modélisation énergétique et optimisation, ORDECSYS, 1224 Chêne-Bougeries, Suisse \\ ${ }^{4}$ Consultante/chercheure en modélisation énergétique, climat et développement, ENERIS, 28012 Madrid, Espagne
}

\begin{abstract}
Les modèles technoéconomiques ont été développés pour représenter l'interaction entre le système économique général et les innovations technologiques dans un secteur particulier, très souvent dans le domaine de l'énergie. Leur objectif est de proposer des solutions « optimales » d'itinéraires technoéconomiques. Au niveau mondial, ces modèles intégrés, tout en tenant compte d'une hétérogénéité macro-régionale, apparaissent comme des représentations utiles pour tester différents choix de développement (IMAGE néerlandais, IMACLIM-R français, par exemple). À partir du modèle ETEM-AR, cousin des précédents, les auteurs proposent une démarche dont l'originalité est triple. Tout d'abord, le modèle est élaboré au niveau régional (la région Midi-Pyrénées après d'autres expérimentations de l'équipe), ce qui pose des questions de mobilisation des données régionales et éventuellement de mesures des échanges avec les autres régions françaises. Ils combinent ensuite les scénarios étudiés de l'atténuation et de l'adaptation au changement climatique. Enfin, ils prennent en compte de façon explicite les incertitudes, les vulnérabilités et les risques liés au changement climatique, en déployant des méthodes de résolution adaptées, stochastiques et robustes. Les solutions robustes sont constituées d'un panier (mix) de technologies qui permet une meilleure couverture des risques. On peut espérer de ces approches une façon de mieux raisonner les composantes d'une politique énergétique régionalisée, ce qui appelle une réelle incorporation des acteurs locaux dans la démarche de modélisation.
\end{abstract}

La Rédaction

\section{Mots-clés :}

environnement : changement climatique ; politiques climatiques; gestion de l'incertain ; modélisation

\begin{abstract}
Résumé - Cet article présente le modèle d'optimisation technico-économique ETEM-AR consacré à l'analyse du futur d'un système de production et d'utilisation de l'énergie à l'échelle régionale. Le modèle prend en compte aussi bien l'atténuation de l'émission de gaz à effet de serre que l'adaptation aux impacts du changement climatique. L'article montre qu'il est possible de combiner l'analyse des politiques d'atténuation et celle des politiques d'adaptation dans des modèles technico-économiques du système énergétique. Le recours à la simulation de scénarios contrastés, à la programmation stochastique et à l'optimisation robuste, permet de prendre en compte les incertitudes associées aux caractéristiques du système énergétique et aux impacts futurs du changement climatique. Le modèle est illustré par une application à la région française Midi-Pyrénées.
\end{abstract}

Auteur correspondant : F. Babonneau, fbabonneau@ordecsys.com Alain Haurie est professeur honoraire à l'Université de Genève (Suisse). Frédéric Babonneau est également collaborateur scientifique au LEURE à l'École polytechnique fédérale de Lausanne (Suisse). 


\section{Keywords:}

environment; climate

change; climate

policies; uncertainty

management;

prospective modelling climate change and of its consequences on the energy sector are treated via stochastic programming and robust optimization. A set of contrasted scenarios is used to describe the possible future consequences of climate change on key parameters of the energy system, such as the reduction of the efficiency of some of the technologies. By comparing the results emerging from the stochastic analysis performed with these scenarios with the analysis of deterministic scenarios, one can understand the necessity, or the absence of it, of investing in certain adaptation technologies prior to the unfolding of the considered uncertainties. The robust optimization techniques are used to ensure the optimal strategy remains a good strategy if some parameters vary in a given interval. The main effect of using a robust analysis is the emergence of a diversification of the technology choices. The model, ETEM-AR, is illustrated here through its application to the French region of Midi-Pyrénées.

\section{Introduction}

Dans le but d'aider les acteurs et les décideurs régionaux à identifier les mesures d'atténuation et d'adaptation appropriées dans le secteur de l'énergie, le modèle ETEM-AR ${ }^{1}$ a été développé et testé lors d'une application pilote au cas de la région Midi-Pyrénées ${ }^{2}$, en France. ETEM-AR est un modèle technico-économique dynamique qui permet d'évaluer de manière combinée les mesures d'atténuation et d'adaptation. Il prend en compte les mesures de réduction des émissions de gaz à effet de serre ainsi que celles d'adaptation (A) nécessaires pour pallier la vulnérabilité climatique des activités propres au système énergétique. Avec ce modèle, nous formulons divers problèmes d'optimisation selon la nature des incertitudes. Leurs solutions forment un choix de filières technologiques et énergétiques qui est robuste (R), c'est-à-dire qui demeure adapté pour un ensemble de scénarios contrastés concernant les mesures de réduction des émissions de gaz à effet de serre qui seront décidées, la disponibilité ou le coût des nouvelles technologies ainsi que les impacts du changement climatique à venir sur les demandes de services énergétiques et sur l'efficacité de certaines technologies de production d'électricité.

L'analyse des mesures d'atténuation dans le secteur de l'énergie s'est déjà effectuée à l'aide de modèles technico-économiques tels que MARKAL, TIMES, MESSAGE, OSeMOSYS, ETEM ${ }^{3}$, ou de modèles intégrés tels que TIAM-WORLD ${ }^{4}$. Lorsque l'on traite aussi de l'adaptation, il est d'usage de distinguer les mesures d'adaptation proactive, qui sont des investissements fournissant

\footnotetext{
1 Il s'agit d'une évolution du modèle ETEM distribué en mode open source par la société ORDECSYS, www.ordecsys. com.

2 Les résultats présentés dans cet article ont une valeur exploratoire et ne représentent aucunement des souhaits ou recommandations politiques pour la région Midi-Pyrénées.

3 ETEM est un modèle inspiré du modèle MARKAL et conçu pour faciliter la modélisation énergétique au niveau des régions ou des villes (cantons en Suisse).

4 TIAM-WORLD inclut aussi un module décrivant l'évolution du climat (Loulou et Labriet, 2008).
}

une couverture des risques, et les mesures d'adaptation réactive, qui sont prises pour pallier les effets du changement climatique au moment où ils se manifestent. L'analyse des mesures d'atténuation doit tenir compte des incertitudes concernant la mise en place de politiques internationales de réduction des émissions de gaz à effet de serre ainsi que la disponibilité et le coût de nouvelles technologies. L'analyse des mesures d'adaptation dans le secteur de l'énergie se heurte notamment aux difficultés épistémologiques mises en évidence par Dessai et Hulme $(2004,2007)$. Ces auteurs montrent que l'incertitude qui subsiste dans les modèles climatiques est trop grande, en particulier au niveau des impacts régionaux, pour que l'on puisse envisager une approche où on prédirait l'évolution climatique, les impacts possibles et où l'on définirait, ensuite, les mesures d'adaptation les plus efficaces. Ces auteurs, associés à Lempert et Pielke (Dessai et al., 2009), proposent alors une approche basée sur la recherche de "politiques robustes ». Dans la modélisation technico-économique ETEM-AR, s'appuyant sur un paradigme d'optimisation, la recherche de politiques robustes s'effectue par la prise en compte des principales sources d'incertitude sur les paramètres du modèle au moyen des techniques de la programmation stochastique et de l'optimisation robuste.

L'analyse par scénarios en programmation stochastique a déjà été largement utilisée dans les modèles de type MARKAL. Une première mise en œuvre a été effectuée dans Fragnière et Haurie (1996) pour un modèle régional correspondant au canton de Genève. Les modèles TIMES et TIAM-WORLD ont été programmés pour pouvoir facilement appliquer une programmation stochastique à deux périodes (Loulou et al., 2009). Le modèle ETEM a été complété par le logiciel Det2Sto qui permet de générer automatiquement une version sous forme extensive d'un programme stochastique multipériodes, à partir de données décrivant les différents scénarios possibles (Thénie et al., 2007). La recherche de politiques robustes dans des modèles technico-économiques a été réalisée en utilisant le critère dit de minimax regret (Loulou et Kanudia, 1999), et, dernièrement, en mettant en œuvre les techniques récemment développées de l'optimisation robuste (Babonneau et al., 2012). Les succès 
remportés lors de l'introduction de ces méthodes d'optimisation robuste dans des modèles technico-économiques orientés vers l'atténuation nous invitent donc à explorer la possibilité d'inclure par ce biais l'analyse de l'adaptation dans les modèles technico-économiques de systèmes énergétiques régionaux basés sur un paradigme d'optimisation et à proposer ainsi un outil opérationnel pouvant aider à l'établissement de plans climat territoriaux. Une application pilote dans la région MidiPyrénées a été réalisée grâce à la collaboration de $1^{\prime}$ Oremip ${ }^{5}$. L'accent a été mis sur les variations possibles dans les demandes utiles, en particulier celles reliées au chauffage et à la climatisation des bâtiments, ainsi que sur la vulnérabilité climatique de différentes technologies de production d'électricité.

Dans le reste de cet article, nous présentons, en deuxième partie, la structure des modèles technico-économiques utilisés et nous discutons de l'introduction des deux approches combinées pour traiter de l'incertitude dans les processus d'adaptation au changement climatique ; en troisième partie, nous exposons le scénario de référence pour la région Midi-Pyrénées qui correspond à une situation d'anticipation parfaite, sans effets notoires dus au changement climatique; en quatrième partie, nous présentons la solution obtenue lorsque l'on introduit les incertitudes correspondant aux impacts possibles du changement climatique dans la modélisation; en cinquième partie, nous tirons les enseignements de cet exercice ; enfin, en conclusion, nous abordons la question de l'utilisation possible de cette approche lors de l'élaboration de plans climat territoriaux. Une annexe mathématique résume les approches d'optimisation mises en œuvre dans cette étude.

\section{Modélisation robuste de l'atténuation et de l'adaptation dans un système énergétique régional}

Dans cette partie, nous rappelons la structure de programme linéaire des modèles d'optimisation technicoéconomiques déjà utilisés pour analyser les politiques d'atténuation et nous décrivons les approches permettant $\mathrm{d}$ 'introduire la robustesse requise lorsque l'on veut être en mesure d'analyser les politiques d'adaptation. La formulation mathématique du modèle technico-économique ETEM-AR employé dans cette étude est fournie en annexe.

\section{Un modèle de type coût/efficacité en programmation linéaire}

ETEM-AR est un modèle technico-économique permettant une analyse coût-efficacité basée sur l'utilisation de

\footnotetext{
5 Observatoire régional de l'énergie Midi-Pyrénées, http:/ / www.oremip.fr/.
}

la programmation linéaire. La modélisation de l'évolution à longue échéance (horizon de 50 ans ou plus) du système énergétique $d^{\prime}$ une région, $d$ 'une nation ou d'un ensemble de nations échangeant des flux de ressources et d'énergie s'est développée considérablement depuis les années 1980, suite à la création des modèles EFOM (Finon, 1979) et MARKAL (Fishbone et Abilock, 1981), tous deux employant le paradigme de la programmation linéaire pour simuler les mécanismes de marché ${ }^{6}$. Le comité Energy Technology Systems Analysis Program $\left(\right.$ ETSAP $\left.^{7}\right)$ de l'Agence internationale de l'énergie a fait évoluer ces deux modèles vers la modélisation TIMES ${ }^{8}$ qui a été utilisée pour analyser les politiques de l'énergie relatives à l'atténuation des émissions de gaz à effet de serre pour plus de 70 pays ou régions du monde. Pour certains pays, comme la Suède ou la Suisse, qui ont des systèmes énergétiques très décentralisés, des versions du modèle MARKAL adaptées à la modélisation du système énergétique au niveau d'une région ou d'une ville ont été développées à Göteborg (Wene, 1980 ; Wene et Andersson, 1983; Wene et Rydén, 1988), Genève (Fragnière et Haurie, 1996), New York (Hill, 1996). La version de Genève, écrite dans un langage de modélisation libre et appelée MARKAL-LITE a été utilisée en couplage avec des modèles de simulation de la pollution de l'air (Haurie et al., 2004). Une application récente de ETEM à l'analyse de l'impact des réseaux intelligents (smart grids) associés aux énergies renouvelables intermittentes est présentée dans Babonneau et al. (2012).

Tous ces modèles partagent la même structure. Tout d'abord, ce sont des modèles d'expansion de capacité qui représentent les investissements dans divers types de technologies d'extraction, de production, de transformation et d'usage des différentes formes d'énergie. Ces investissements créent des capacités de production pour ces technologies qui sont des transformateurs de ressources, en particulier de ressources d'énergie. Les formes d'énergie considérées sont tout d'abord les ressources primaires et les importations, puis, par l'usage des technologies installées, on transforme ces énergies primaires en énergies secondaires puis en énergies utiles, c'est-à-dire en énergie servant à satisfaire des prestations de demande telles que le transport, individuel ou public, la force motrice et la chaleur industrielle, le chauffage et la climatisation des résidences et des immeubles commerciaux, les usages captifs de l'électricité, etc. Le modèle comptabilise les émissions de rejets polluants et de gaz à effet de serre (la description des technologies polluantes incorpore leur taux d'émission de ces divers rejets). Les technologies sont caractérisées par des durées de vie, des efficacités dans leur

\footnotetext{
6 MARKAL signifie Market allocation.

7 http://www.iea-etsap.org.

8 The Integrated MARKAL-EFOM System (TIMES).
} 

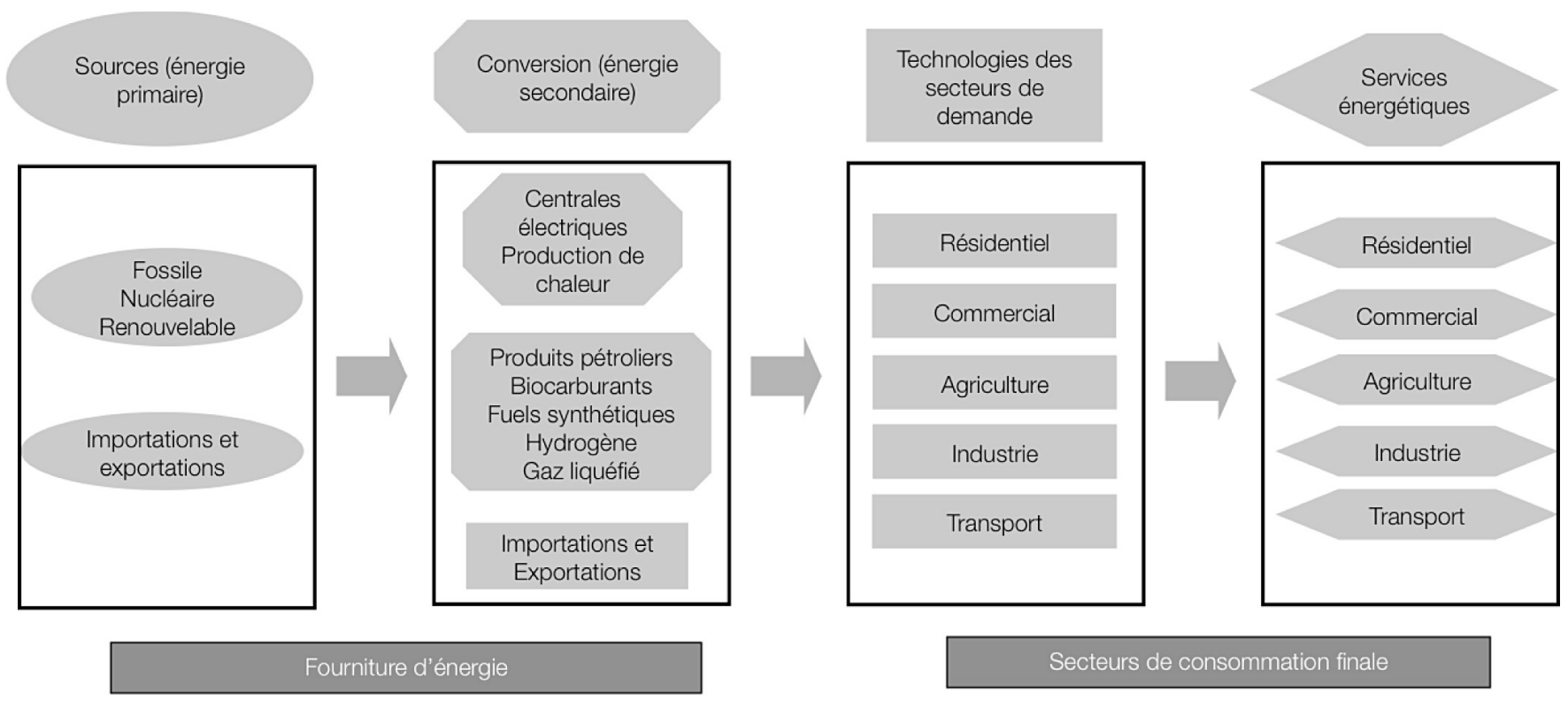

Secteurs de consommation finale

Fig. 1. Système énergétique de référence.

transformation de ressources, par un facteur de disponibilité (typiquement, un panneau solaire ne peut être utilisé qu'une fraction de la journée) et par des paramètres économiques tels que coût de fonctionnement, coût d'entretien, coût d'investissement ainsi que par une date de disponibilité lorsqu'il s'agit de nouvelles technologies. Chaque année est découpée en tranches horaires permettant de tenir compte des variations saisonnières et quotidiennes des demandes d'électricité et de chaleur bassetempérature ainsi que des disponibilités des moyens de production basés sur des sources renouvelables (hydraulique, solaire, éolien, etc.). Le modèle impose aussi de créer une réserve de capacité pour la production d'énergie non stockable, l'électricité en particulier, afin de pouvoir répondre aux demandes de pointe sur certaines tranches horaires. La figure 1 résume la structure du système énergétique de référence qui est modélisé.

Outre ces données technico-économiques concernant les technologies, les informations suivantes doivent être renseignées afin que le modèle soit en mesure de décrire l'évolution du système énergétique d'une région : (i) le prix et la disponibilité des énergies importées; (ii) les capacités déjà installées des diverses technologies et leur durée de vie résiduelle; (iii) les prévisions d'évolution des demandes utiles sur la période de planification (dans certaines mises en œuvre, on introduit une élasticité de chaque demande utile au prix, calculée sur la base du coût marginal des énergies utilisées pour la satisfaire) ; (iv) les bornes sur les émissions de polluants ou de gaz à effet de serre. Un ensemble d'équations va représenter les flux de ressources d'énergie, depuis l'extraction ou l'importation, en passant par les transformations et le transport, puis l'utilisation dans des technologies d'usage (par exemple, les différents types d'automobile pour le transport individuel, les différents types de chauffage des résidences, etc.), pour subvenir finalement aux demandes utiles et aux demandes d'exportation. Un programme linéaire fournit alors une proposition d'évolution de l'organisation du système énergétique dans son ensemble, en optimisant les filières d'énergie qui satisfont à toutes les contraintes, techniques, économiques et environnementales. La référence Loulou et Labriet (2008) décrit très précisement la structure du modèle TIMES. La structure du modèle ETEM est très proche de celle de TIMES ; elle est détaillée dans l'annexe de cet article et dans une application récente pour une région de la Suisse (Babonneau et al., 2012).

\section{Représentation de la vulnérabilité et des risques dans ETEM-AR}

Ainsi que le font remarquer Dessai et Hulme (2007), une étude de l'adaptation au changement climatique est nécessairement effectuée dans un cadre où l'incertitude domine.

\section{Ensembles d'incertitude pour les paramètres du modèle}

L'impact du changement climatique sur le système énergétique d'une région va se refléter dans différents paramètres du modèle. Typiquement, une augmentation des températures va induire des modifications des demandes utiles pour le chauffage et la climatisation des 
bâtiments. Une hausse des températures et une baisse des précipitations peuvent changer les apports d'eau dans les vallées équipées de barrages et de centrales hydroélectriques, et donc changer leur taux de disponibilité. De la même manière, l'augmentation de température peut réduire l'efficacité et la disponibilité des centrales nucléaires. Les technologies basées sur l'utilisation de sources météodépendantes (solaires, éoliennes) peuvent aussi avoir, de ce fait, des efficacités et des disponibilités incertaines, surtout à long terme. Une première étape dans la représentation des vulnérabilités climatiques consiste donc à identifier des plages de variations pour certains paramètres relatifs aux demandes utiles, aux efficacités et aux disponibilités des technologies. D'autres incertitudes qui concernent les prix et les disponibilités des formes d'énergie importées (pétrole et gaz, par exemple) peuvent aussi être prises en compte, de même que l'évolution possible des politiques environnementales, en particulier en ce qui concerne la réduction imposée des émissions de gaz à effet de serre.

\section{Scénarios contrastés}

Pour faire apparaître des politiques d'adaptation au changement climatique, nous analysons différents scénarios où certains paramètres cruciaux prennent des valeurs extrêmes dans leur ensemble d'incertitude. Nous appliquons notamment cette méthode à la modification de la demande en chauffage et en refroidissement des bâtiments en Midi-Pyrénées.

\section{Scénarios stochastiques}

Pour analyser l'intérêt de politiques d'adaptation proactive par rapport à des politiques réactives, une approche de programmation stochastique peut être utilisée. Plusieurs scénarios sont alors représentés par les différents chemins dans un arbre d'événements. Les premières décisions, prises à la source de l'arbre d'événements, sont communes à tous les scénarios ; elles correspondront à des mesures de couverture, autrement dit à des adaptations préventives ou proactives ; les décisions prises ensuite dépendent du scénario qui se réalise et sont communément appelées variables de recours en recherche opérationnelle. Elles sont les adaptations réactives. Dans notre modèle qui comporte de nombreuses variables et contraintes, la mise en œuvre de la programmation stochastique est grandement facilitée par l'utilisation de l'outil logiciel Det2Sto (Thénie et al., 20079). Une description mathématique de la mise en œuvre de la programmation stochastique employée dans cette étude se trouve en annexe.

\section{Optimisation robuste}

Les méthodes de l'optimisation robuste (Ben-Tal et al., 2009) fournissent une alternative permettant de prendre en compte l'incertitude des paramètres du modèle. L'optimisation robuste propose de générer un modèle mathématique traitable au sens de la théorie de la complexité en optimisation et en faisant appel le moins possible à des concepts probabilistes. L'approche robuste reconnaît qu'il est en général impossible de se prémunir totalement contre une violation de contrainte. Son objectif est de ne l'admettre que dans des cas rares, peu pertinents. L'ensemble d'incertitude rassemble les valeurs des paramètres incertains jugés pertinents pour la contrainte. Une solution est dite robuste vis-à-vis d'une contrainte si cette dernière est satisfaite pour toute réalisation dans l'ensemble d'incertitude. Bien que ne faisant appel à aucun concept de probabilité, l'optimisation robuste induit, sous des conditions très générales, des solutions qui garantissent une probabilité imposée a priori de satisfaction de la contrainte.

Dans cette étude, nous appliquons l'optimisation robuste à l'incertitude des coefficients techniques des nouvelles technologies. Cette incertitude est liée aux impacts du changement climatique, mais aussi aux résultats incertains de la recherche et développement en ce qui concerne les nouvelles technologies. À première vue, l'optimisation robuste peut être assimilée à une approche qui se prémunit contre la pire situation pour un sousensemble de réalisations des aléas. Cette approche a deux avantages cruciaux: (i) elle ne fait pas appel à des concepts probabilistes; (ii) elle n'induit sur le modèle déterministe original que des modifications limitées en termes d'ajout de variables et de contraintes. Une description mathématique de la mise en œuvre des méthodes d'optimisation utilisées dans cette étude est fournie en annexe. Dans l'application numérique que nous verrons plus loin, nous employons l'optimisation robuste pour prendre en compte les incertitudes relatives à la disponibilité des éoliennes, des centrales nucléaires et hydrauliques et relatives aux coûts des technologies futures.

En combinant, d'un côté, la programmation stochastique pour les demandes en climatisation et en chauffage, et, de l'autre, l'optimisation robuste pour intégrer les incertitudes de disponibilités et de coûts, l'on propose des solutions de recours sur chacune des branches de l'arbre de décision qui s'appuient sur les réalisations extrêmes des ensembles d'incertitude. En d'autres termes, les adaptations proactives de première période seront dites « robustes », car bonnes pour l'ensemble des scénarios de

\footnotetext{
9 Développé par ORDECSYS et distribué en mode open source, téléchargeable à l'adresse suivante : http:/ / apps.ordecsys.com/det2sto.
} 
l'arbre d'événements et des réalisations des ensembles d'incertitude.

\section{Application à la région Midi-Pyrénées}

Nous présentons dans cette partie une application du modèle à la région Midi-Pyrénées ainsi qu'une sélection des principaux résultats obtenus ${ }^{10}$.

\section{Le système énergétique de la région Midi-Pyrénées (Oremip, 2013)}

En 2011, Midi-Pyrénées compte près de 3 millions d'habitants. La consommation d'énergie finale est de 5,7 millions de tep ${ }^{11}$ en 2011, pour une consommation énergétique par habitant de l'ordre de 2 tep/habitant, plus faible que la moyenne française, $\mathrm{du}$ fait d'une industrie peu énergivore. Les émissions de $\mathrm{CO}_{2}$, en baisse, atteignent $4 \mathrm{t}$ /habitant et sont inférieures à la moyenne française du fait de la structure de l'économie de la région. C'est le secteur des transports qui contribue le plus aux émissions de $\mathrm{CO}_{2}$, reflétant la structure et les consommations énergétiques sectorielles de la région.

Les secteurs résidentiel et tertiaire représentent $44 \%$ de la consommation d'énergie finale (régression progressive des produits pétroliers; le gaz naturel tend à progresser, l'électricité, quant à elle, tend à baisser sous l'effet d'appareils plus efficaces, et le bois se maintient), les transports, $37 \%$ (large domination du diesel), l'industrie, $15 \%$ (domination du gaz, de l'électricité et de la biomasse) et finalement l'agriculture, $4 \%$. L'arrêt des activités des usines AZF en 2001 (à Toulouse), puis des installations Péchiney à partir de 2007 (à Lannemezan, dans les Hautes-Pyrénées) ont contribué à la diminution de la consommation énergétique de l'industrie. Les produits pétroliers représentent $43 \%$ des consommations finales, suivis par l'électricité (25\%), le gaz naturel (16\%) et le bois (11\%).

La production d'électricité d'environ 29 TWh est dominée par le nucléaire (67 \%, centrale de Golfech). Viennent ensuitel'hydraulique (27\%, deux châteaux d'eau : le Massif central et les Pyrénées), puis l'éolien (3\%), le solaire, les centrales thermiques renouvelables - bois, biogaz, déchets - et, enfin, les centrales thermiques fossiles (1\%). Les énergies renouvelables sont en rapide augmentation. Midi-Pyrénées compte plus de 70 grandes centrales (puissance $>8 \mathrm{MW}$ ), représentant $80 \%$ de la puissance hydroélectrique installée, et environ 600 petites centrales (puissance $<8 \mathrm{MW}$ ). Midi-Pyrénées dispose par ailleurs de la troisième forêt de France, et la consommation de bois de chauffage par les ménages correspond à la majorité de la consommation régionale de bois-énergie. En 2010, Midi-Pyrénées comptait 165 éoliennes (soit une puissance installée de $320 \mathrm{MW}$ ) et la puissance raccordée

\footnotetext{
${ }^{10}$ Résultats détaillés sur http:/ / etem-ar.ordecsys.com/.

11 Tonne équivalent pétrole.
}

en photovoltaïque atteignait $80 \mathrm{MW}$ (contre $873 \mathrm{MW}$ pour toute la France). Une superficie de $160000 \mathrm{~m}^{2}$ de panneaux solaires, utilisés pour le chauffage collectif et particulier, était recensée en Midi-Pyrénées en 2011.

\section{La vulnérabilité du système énergétique}

Le Schéma régional climat air énergie (SRCAE) de la région Midi-Pyrénées 2011 identifie les éléments les plus vulnérables du système énergétique de la région MidiPyrénées face aux changements climatiques. Cette analyse permet de caractériser les vulnérabilités les plus importantes à modéliser dans ETEM-AR.

La production d'hydroélectricité est concentrée au niveau des Pyrénées et du Massif central, qui sont les territoires potentiellement les moins affectés par les impacts du changement climatique sur la disponibilité de la ressource en eau.

Concernant le nucléaire, l'une des conséquences directes du changement climatique est le risque de dépassement des températures maximales de rejet $\left(28^{\circ} \mathrm{C}\right)$ en raison du réchauffement de l'eau. La centrale de Golfech a déjà bénéficié $\mathrm{d}$ 'autorisations exceptionnelles de rejet en 2003 (14 jours), 2004 et 2005 afin de ne pas interrompre la production d'électricité. Linnerud et al. (2011) estiment qu'une augmentation de $1{ }^{\circ} \mathrm{C}$ pourrait réduire la production d'une centrale nucléaire de $0,5 \%$ à $2,3 \%$ à cause de la baisse d'efficacité thermique et des arrêts plus fréquents dus aux périodes extrêmes de sécheresse ou de vagues de chaleur.

Sur le plan des demandes, une diminution des consommations d'énergie en hiver et une hausse des consommations en été (augmentation de la température moyenne, mais aussi des vagues de chaleur ${ }^{12}$ ) est anticipée, ce qui devrait se traduire par une nouvelle définition de la courbe de charge, avec des pics de demandes en partie déplacés de l'hiver à l'été. L'estimation des nouvelles demandes de chauffage et de climatisation repose sur le calcul des degrés-jours de chauffage et de climatisation, tels que définis par les modèles climatiques. En lien avec ces variations, le taux d'équipement des ménages en climatiseurs pourrait fortement augmenter. Il est actuellement faible (5\%) en comparaison des pays limitrophes connaissant aujourd'hui un climat plus chaud (25\% en Italie, $36 \%$ en Espagne).

Les tempêtes pourraient avoir des impacts sur les infrastructures de transport et la distribution de l'électricité, les coupures étant principalement liées aux chutes d'arbres. Les options d'adaptation disponibles qui peuvent être modélisées sont la possibilité d'investir dans des

\footnotetext{
12 En 2003, la canicule a mené à une hausse de consommation annuelle d'électricité de 5 à $10 \%$ à l'échelle française, en raison de la sollicitation plus importante des réfrigérateurs, congélateurs, climatiseurs, ventilateurs et instruments industriels de refroidissement.
} 

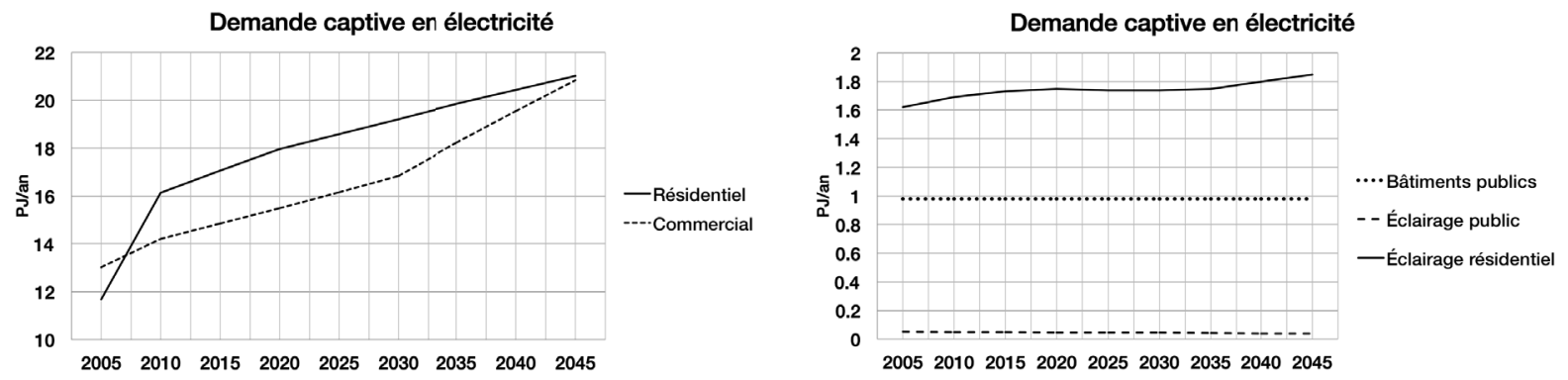

Fig. 2. Demandes utiles en électricité captive (en pétajoules/an).
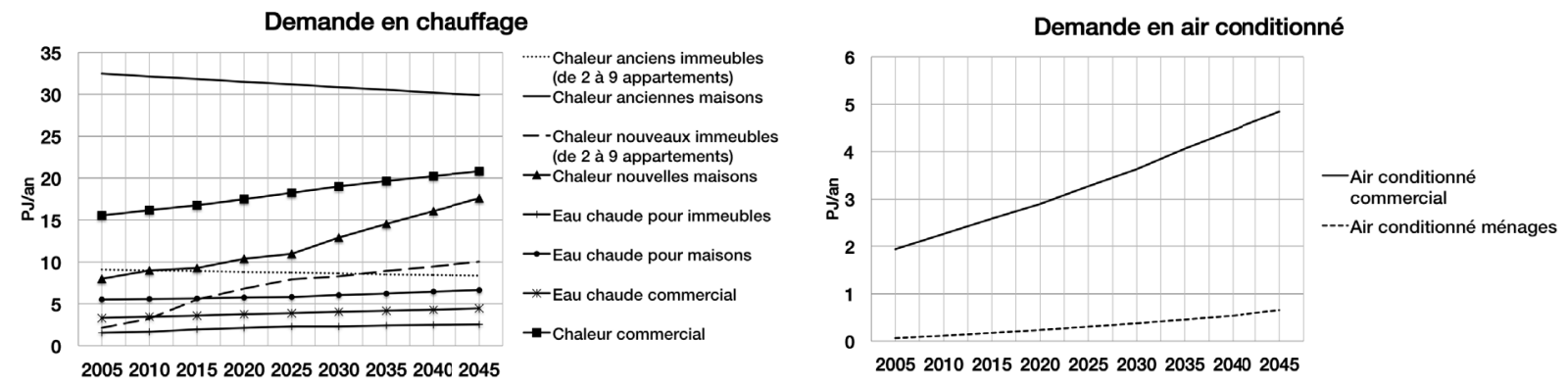

Fig. 3. Demandes utiles en chauffage et en air conditionné (en pétajoules/an).
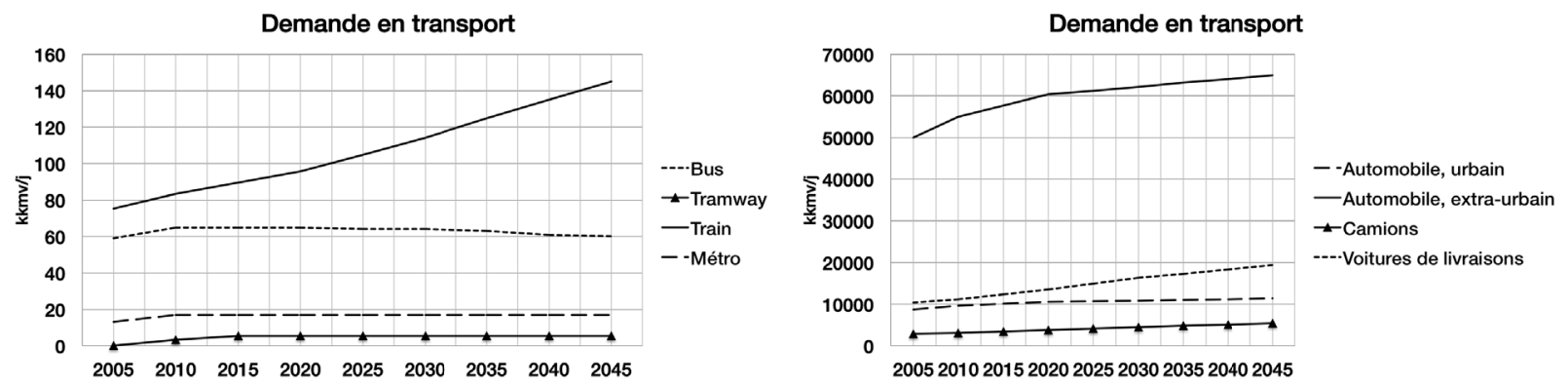

Fig. 4. Demandes utiles en transport public et privé (en milliers de km/véhicule/jour).

réseaux souterrains, celle de minimiser l'utilisation $\mathrm{du}$ réseau haute tension (autrement dit de privilégier la production décentralisée).

L'augmentation des pertes techniques sur le réseau de transport et de distribution n'est pas mentionnée par le SRCAE.

D'autres conséquences sont envisagées, telles que: l'augmentation importante du nombre de piscines individuelles, pouvant être à l'origine d'une hausse de l'utilisation de l'eau et de l'énergie en période d'été ; la surconsommation de carburant liée à la climatisation dans les systèmes de transport. D'autres effets, que ne mentionne pas le SRCAE, tels qu'une demande croissante d'électricité pour produire de la neige artificielle, pourraient à long terme être observés sur l'activité touristique de la région.

À ce jour, aucune information n'existe sur les impacts potentiels des changements climatiques sur les ressources renouvelables autres que l'hydroélectricité et sur la dis- ponibilité locale en bois. Étant donné la part importante du bois dans les consommations résidentielles de la région, l'obtention d'informations complémentaires sur sa disponibilité, à moyen et long terme, est en cours.

\section{Données de base}

Le modèle du système énergétique est défini à partir de données de base constituées des prévisions d'évolution des demandes en services énergétiques (demandes utiles), des prix des énergies importées dans la région et d'une base de données techniques et économiques concernant les différents processus de production et de transformation des formes d'énergie.

\section{Demandes utiles}

Les figures 2 à 4 et le tableau 1 indiquent les évolutions supposées des demandes de services énergétiques dans différents secteurs del'économie dela région Midi-Pyrénées. 
Tableau 1. Demande utile industrielle et autre (pétajoules/an).

\begin{tabular}{|c|c|c|c|c|c|c|}
\hline & & 2005 & 2015 & 2025 & 2035 & 2045 \\
\hline \multirow{3}{*}{ Métallurgie } & chaleur & 3.76 & 4.25 & 4.54 & 4.68 & 4.82 \\
\hline & force motrice & 4.20 & 4.75 & 5.07 & 5.22 & 5.38 \\
\hline & autre & 0.84 & 0.95 & 1.01 & 1.04 & 1.07 \\
\hline \multirow{3}{*}{ Chimie } & chaleur & 4.41 & 4.99 & 5.37 & 5.59 & 5.76 \\
\hline & force motrice & 2.45 & 2.77 & 2.98 & 3.10 & 3.20 \\
\hline & autre & 1.07 & 1.21 & 1.31 & 1.36 & 1.40 \\
\hline \multirow{3}{*}{ Papier } & chaleur & 6.86 & 7.87 & 8.82 & 9.62 & 10.37 \\
\hline & force motrice & 1.04 & 1.19 & 1.33 & 1.46 & 1.57 \\
\hline & autre & 0.68 & 0.79 & 0.88 & 0.96 & 1.04 \\
\hline \multirow{3}{*}{$\begin{array}{l}\text { Matériaux de } \\
\text { construction }\end{array}$} & chaleur & 6.69 & 7.67 & 8.60 & 9.39 & 10.11 \\
\hline & force motrice & 1.61 & 1.85 & 2.07 & 2.26 & 2.44 \\
\hline & autre & 1.70 & 1.95 & 2.19 & 2.39 & 2.57 \\
\hline \multirow{3}{*}{ Autres industries } & chaleur & 0.50 & 0.62 & 0.73 & 0.85 & 0.99 \\
\hline & force motrice & 0.55 & 0.68 & 0.81 & 0.94 & 1.09 \\
\hline & autre & 0.22 & 0.27 & 0.32 & 0.38 & 0.44 \\
\hline \multirow{3}{*}{ Agroalimentaire } & chaleur & 3.11 & 3.84 & 4.57 & 5.30 & 6.15 \\
\hline & force motrice & 1.01 & 1.25 & 1.49 & 1.73 & 2.00 \\
\hline & autre & 0.41 & 0.51 & 0.61 & 0.71 & 0.82 \\
\hline Agriculture & & 9.46 & 9.46 & 9.46 & 9.46 & 9.46 \\
\hline
\end{tabular}

Tableau 2. Prix des énergies importées (en $€ /$ Gigajoule).

\begin{tabular}{|l|c|c|c|c|c|}
\hline & $\mathbf{2 0 0 5}$ & $\mathbf{2 0 1 5}$ & $\mathbf{2 0 2 5}$ & $\mathbf{2 0 3 5}$ & $\mathbf{2 0 4 5}$ \\
\hline Charbon & 3.3 & 5.6 & 6.1 & 6.4 & 21.4 \\
\hline Fioul domestique & 10.5 & 16.2 & 19.5 & 10.4 & 10.8 \\
\hline Fioul lourd & 5.9 & 9.2 & 9.9 & 26.0 & 27.8 \\
\hline Diesel pour transport & 13.2 & 19.7 & 23.6 & 25.5 & 27.3 \\
\hline Essence sans plomb & 11.5 & 19.3 & 23.2 & 40.0 & 42.0 \\
\hline Hydrogène & 30.0 & 34.0 & 38.0 & 57.4 & 61.5 \\
\hline Méthanol & 28.0 & 43.6 & 52.2 & 16.9 & 18.8 \\
\hline Gaz naturel & 7.2 & 12.7 & 15.4 & 18.9 & 20.8 \\
\hline Biogaz & 9.2 & 14.7 & 17.4 & 16.0 & 20.0 \\
\hline Biomasse & 10.0 & 11.3 & 13.5 & 0.1 & 0.1 \\
\hline Déchets ménagers valorisables & 0.1 & 0.1 & 0.1 & 24.2 & 26.0 \\
\hline Gaz de pétrole liquéfié & 11.2 & 17.9 & 21.8 & 28.5 & 30.3 \\
\hline Bioéthanol & 20.0 & 20.0 & 26.2 & 29.0 & 30.8 \\
\hline Biodiesel & 20.0 & 20.0 & 26.6 & 30.9 & 36.4 \\
\hline Électricité (jour) & 23.7 & 13.2 & 13.2 & 15.2 & 17.2 \\
\hline Électricité (nuit) & & & 20.2 \\
\hline
\end{tabular}

\section{Prix des formes d'énergie importées}

Le tableau 2 indique les hypothèses d'évolution des prix des différentes formes d'énergie importées dans la région Midi-Pyrénées.

\section{Données technologiques}

La base de données technologiques est adaptée de celle des modèles MARKAL/TIMES et peut être consultée sur le site du projet ${ }^{13}$.

\footnotetext{
13 http:/ / etem-ar.ordecsys.com.
} 


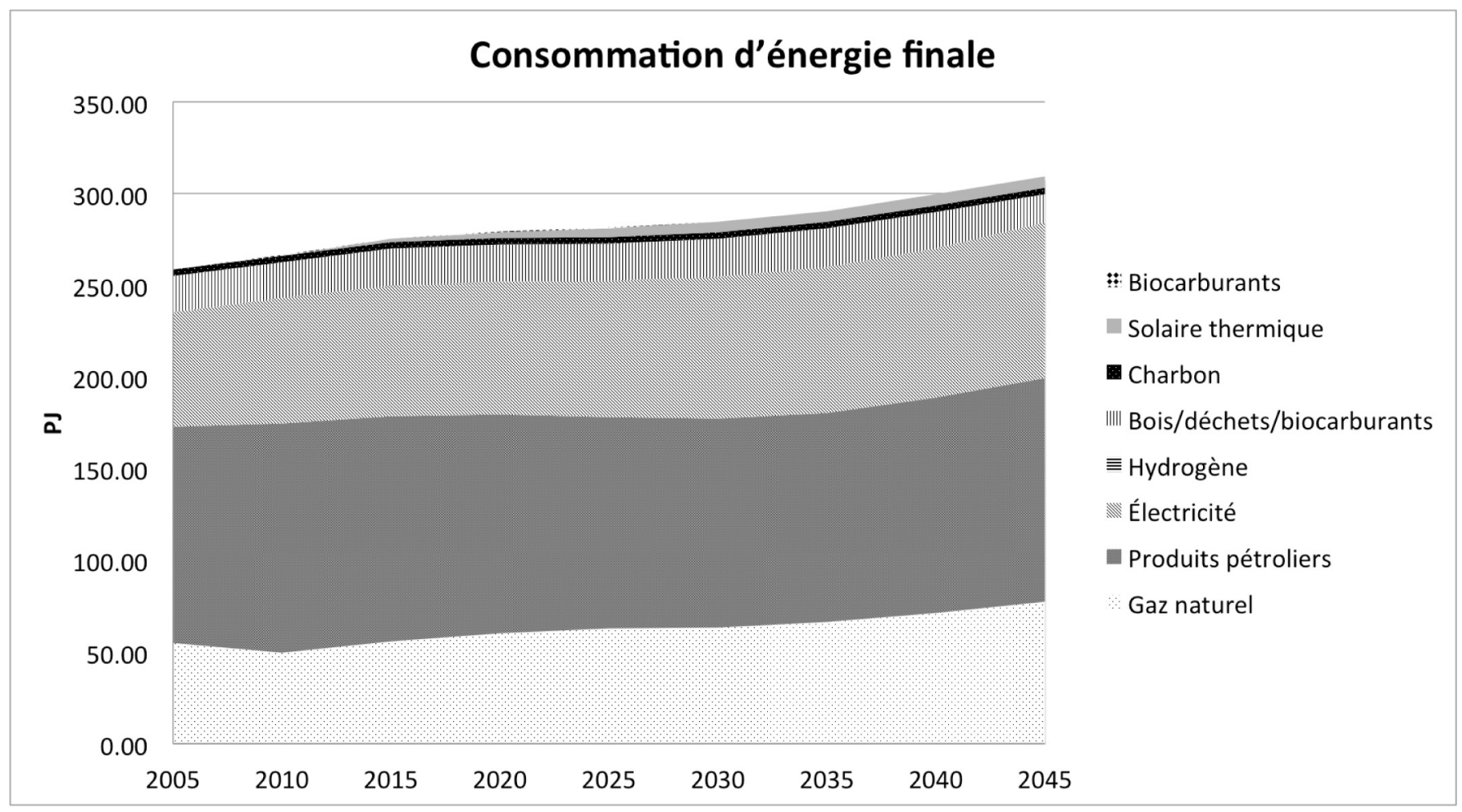

Fig. 5. Consommations par forme d'énergie finale (en pétajoules).

\section{Analyse prospective du scénario de référence}

Dans le scénario de référence, aucune contrainte sur les émissions de gaz à effet de serre n'est imposée et aucune incertitude induite par le changement climatique n'est introduite. En optimisant le coût systémique total des coûts d'importation, de production et d'investissement constitué de la somme actualisée au taux annuel de $3 \%$, on obtient le profil prospectif suivant pour l'évolution des choix énergétiques et technologiques dans la région.

\section{Évolution des bilans énergétiques}

La figure 5 indique l'évolution des consommations d'énergie finale à l'horizon 2045. On constate une croissance de la consommation totale et une relative stabilité dans les proportions relatives des différentes formes d'énergie finale dans le bilan énergétique.

\section{Choix technologiques}

- Chauffage : Concernant l'activité des technologies de chauffage, on observe une nette domination des systèmes au gaz.

- Transport: On observe une hégémonie des véhicules hybrides pour le transport individuel urbain et extraurbain (Fig. 9, en haut à gauche) et des véhicules Diesel pour le transport commercial.
- Production d'électricité : On observe une légère croissance de la production éolienne et des petites unités hydroélectriques (Fig. 10, en haut à gauche). Il est important de noter que la région Midi-Pyrénées exporte de l'électricité vers le reste de la France. Dans la modélisation, nous avons imposé que ces exportations demeurent constantes sur tout l'horizon et dans tous les scénarios.

\section{Émissions de gaz à effet de serre}

Ces émissions sont relativement stables à long terme, malgré l'augmentation de la demande en transport en particulier (Fig. 8, en haut à gauche). Cela est dû à la forte pénétration des véhicules hybrides.

\section{Scénario avec adaptation}

Deux des principaux impacts possibles du changement climatique sur le système énergétique sont pris en compte, sur la base des caractéristiques de la région : la modification de la demande utile en climatisation et en chauffage et les incertitudes sur le facteur de disponibilité de certaines technologies de production d'électricité. Par ailleurs, le risque de changement climatique va entraîner une obligation de réduction des émissions de gaz à effet de serre. Dans cette partie, nous introduisons dans le modèle de la région Midi-Pyrénées, une représentation 


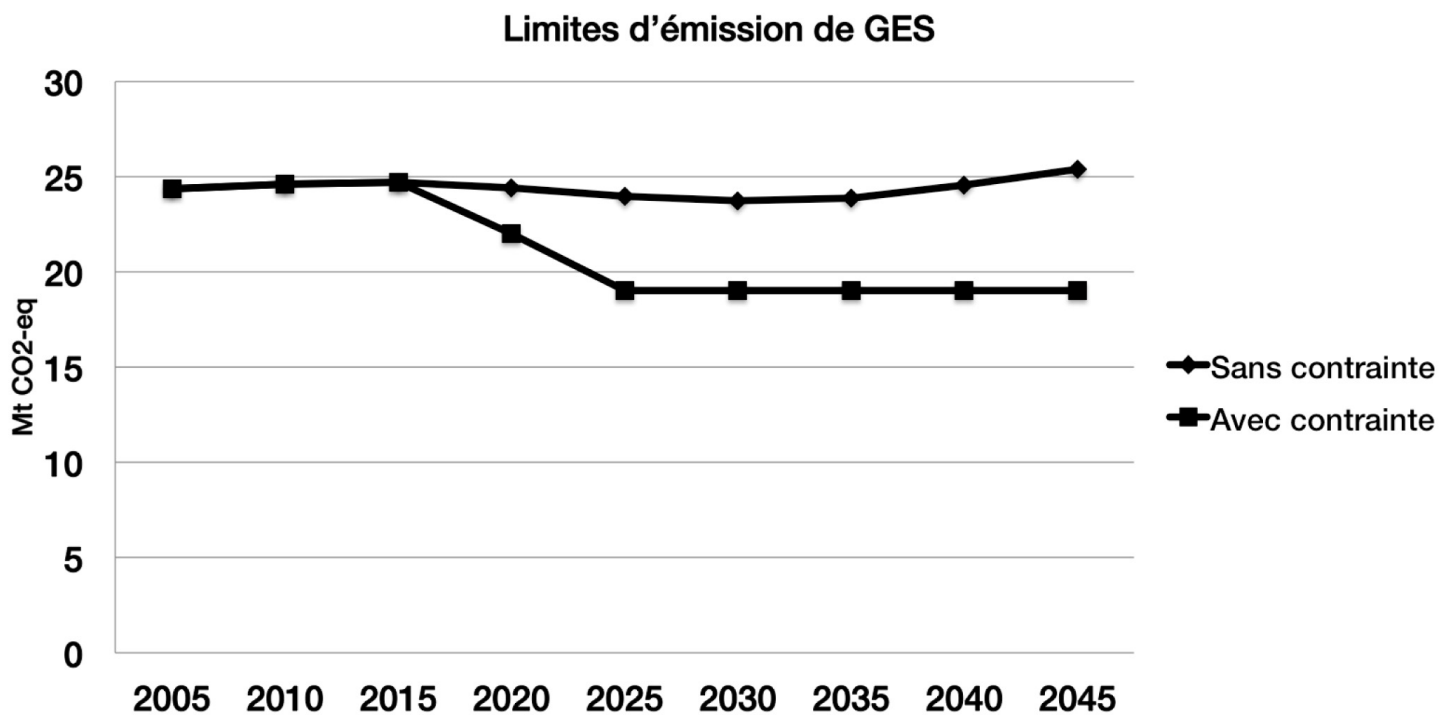

Fig. 6. Profils d'émissions de gaz à effet de serre avec et sans contrainte environnementale $\left(\mathrm{Mt}^{\mathrm{CO}} \mathrm{O}_{2}\right.$ eq. $)$.

des principales incertitudes et de l'objectif d'atténuation, et nous procédons à une analyse prospective en utilisant la programmation stochastique et l'optimisation robuste. Nous représentons ci-dessous les principaux secteurs touchés par la prise en compte de l'incertain.

\section{Objectif d'atténuation}

Nous imposons au système énergétique de réduire de $25 \%$ les rejets de gaz à effet de serre à l'horizon 2045 (Fig. 6).

\section{Représentation de l'incertitude}

Afin de modéliser les différentes sources d'incertitude, nous définissons des intervalles de variation possible pour les valeurs de paramètres de disponibilité et pour les coûts de certaines nouvelles technologies (voir infra). Ces intervalles seront exploités, soit dans l'approche d'optimisation robuste afin de définir des ensembles d'incertitude, soit dans la programmation stochastique pour construire un arbre d'événements, comme décrit dans les parties précédentes. Étant donnéle type d'analyse effectuée (horizon d'étude 2005-2050 et pour une région spécifique du sud de la France), les incertitudes dominantes sont celles liées aux modèles climatiques, entre $60 \%$ et $80 \%$ de l'incertitude totale, tandis que la part d'incertitude liée au scénario est limitée entre $0 \%$ et $20 \%$ (Hawkins et Sutton, 2009). Dans le cadre de cette étude, l'évaluation des incertitudes provenant du changement climatique résulte d'une analyse détaillée de simulations de plusieurs modèles climatiques (ARPEGE, HADGEM2, IPCM4, CNCM33, DMIEH5C, MPEH5C) pour le scénario climatique A1B décrit dans le rapport du GIEC sur les scénarios d'émissions. En particulier, nous avons déterminé l'évolution des températures et des précipitations pour chacun des modèles.

\section{Besoins de climatisation et de chauffage}

L'évolution des températures a servi au calcul des degrés-jours de refroidissement (DJR) et de chauffage (DJC), utilisés pour évaluer les besoins de climatisation et de chauffage en fonction du climat futur. La demande en refroidissement en 2045 est multipliée par 2,5 à 7,2 selon les modèles étudiés ( 4,8 en moyenne), tandis que la demande en chauffage en 2045 est multipliée par 0,7 à $0,9(0,75$ en moyenne) en hiver, et par 0,85 à $0,90(0,90$ en moyenne) en saison intermédiaire. On notera aussi une légère modification de la répartition saisonnière des demandes de chauffage, avec une augmentation de la part de chauffage en hiver et une diminution dans la saison intermédiaire. Cette modification est représentée dans ETEM-AR.

Nous utilisons un arbre d'événements avec trois branches pour représenter l'incertitude concernant l'évolution de la demande utile en chauffage et en climatisation. Le scénario moyen correspond à la moyenne des hausses de la température pour l'ensemble des modèles, alors que le scénario extrême correspond au modèle fournissant l'évolution des températures la plus défavorable. On affecte une probabilité $1 / 3$ à chaque scénario, comme l'indique la figure 7. 


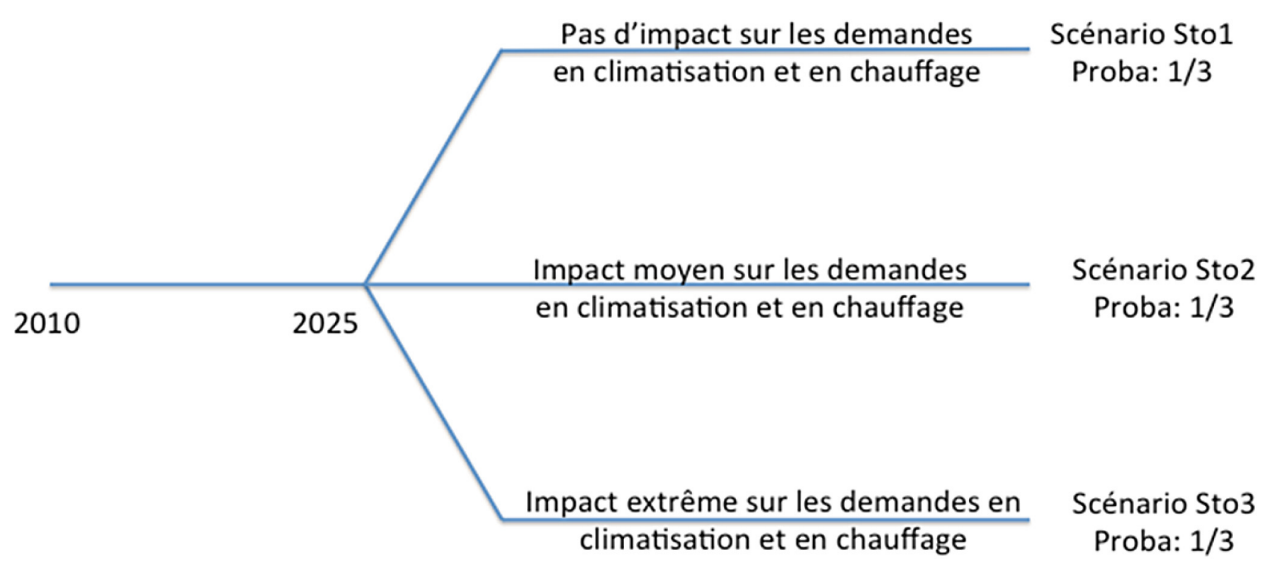

2050

Fig. 7. Arbre d'événements concernant l'impact sur les demandes utiles.

Pour modéliser les sources d'incertitude qui suivent, nous utilisons exclusivement l'optimisation robuste (voir en annexe). Des ensembles d'incertitude sont ainsi définis sur chacune des branches de l'arbre d'événements et l'on cherchera la meilleure solution admissible quelles que soient les réalisations des paramètres incertains dans ces ensembles.

\section{Disponibilité de la centrale nucléaire de Golfech}

L'évaluation de l'amplitude des vagues de chaleur (plus de $30^{\circ} \mathrm{C}$ pendant plus de six jours) a notamment permis d'estimer la baisse possible de la disponibilité de la centrale de Golfech à 7 points de pourcentage au maximum en été. On rappellera que l'une des conséquences directes du changement climatique est le risque de dépassement des températures maximales de rejets $\left(28^{\circ} \mathrm{C}\right)$ en raison du réchauffement de l'eau.

\section{Disponibilité des nouvelles petites centrales hydrauliques}

L'impact des changements climatiques sur la production hydroélectrique est considéré comme incertain et globalement faible dans la région Midi-Pyrénées, car la production se concentre dans les Pyrénées et le Massif central, qui sont les territoires potentiellement les moins affectés par les impacts du changement climatique sur la disponibilité de la ressource en eau.

L'impact conjugué de l'acceptabilité sociale et des possibles conséquences des changements climatiques, estimées à partir des vagues de sécheresse ${ }^{14}$ observées dans les scénarios climatiques, peut rendre indisponible $74 \%$ de la capacité des nouvelles petites unités et réduire de $7 \%$ le facteur de disponibilité en été.

\section{Disponibilité des éoliennes}

L'étude des variations des vents moyens dans différents modèles climatiques ne permet pas de dégager de variation explicite. En outre, un autre facteur d'incertitude important quant à la capacité installée des futures éoliennes tient à l'acceptabilité sociale de ces installations. Une variation de $50 \%$ du facteur de disponibilité a été choisie arbitrairement aux fins du présent exercice, reflétant la possible résistance de la population à l'implantation de nouvelles éoliennes en Midi-Pyrénées.

\section{Coût des installations solaires}

Une incertitude quant au coût des installations destinées à exploiter le potentiel solaire de la région est considérée à partir de 2025 : les coûts d'investissement peuvent ainsi subir une augmentation maximale de $50 \%$.

\section{Analyse prospective}

Nous présentons dans cette partie les résultats de l'optimisation stochastique et robuste et comparons ces résultats à ceux du scénario de référence. Pour chaque point analysé, nous produisons quatre graphiques, le premier correspondant au cas de référence et les trois autres aux trois scénarios de l'arbre d'événements. Rappelons que, dans ces trois derniers scénarios, les valeurs des variables de décision sont forcément identiques pour les périodes 2005 à 2025.

\footnotetext{
${ }^{14}$ Données de sécheresse météorologique : nombre de jours où les précipitations ne dépassent pas $1 \mathrm{~mm} /$ jour durant au moins 6 jours.
} 

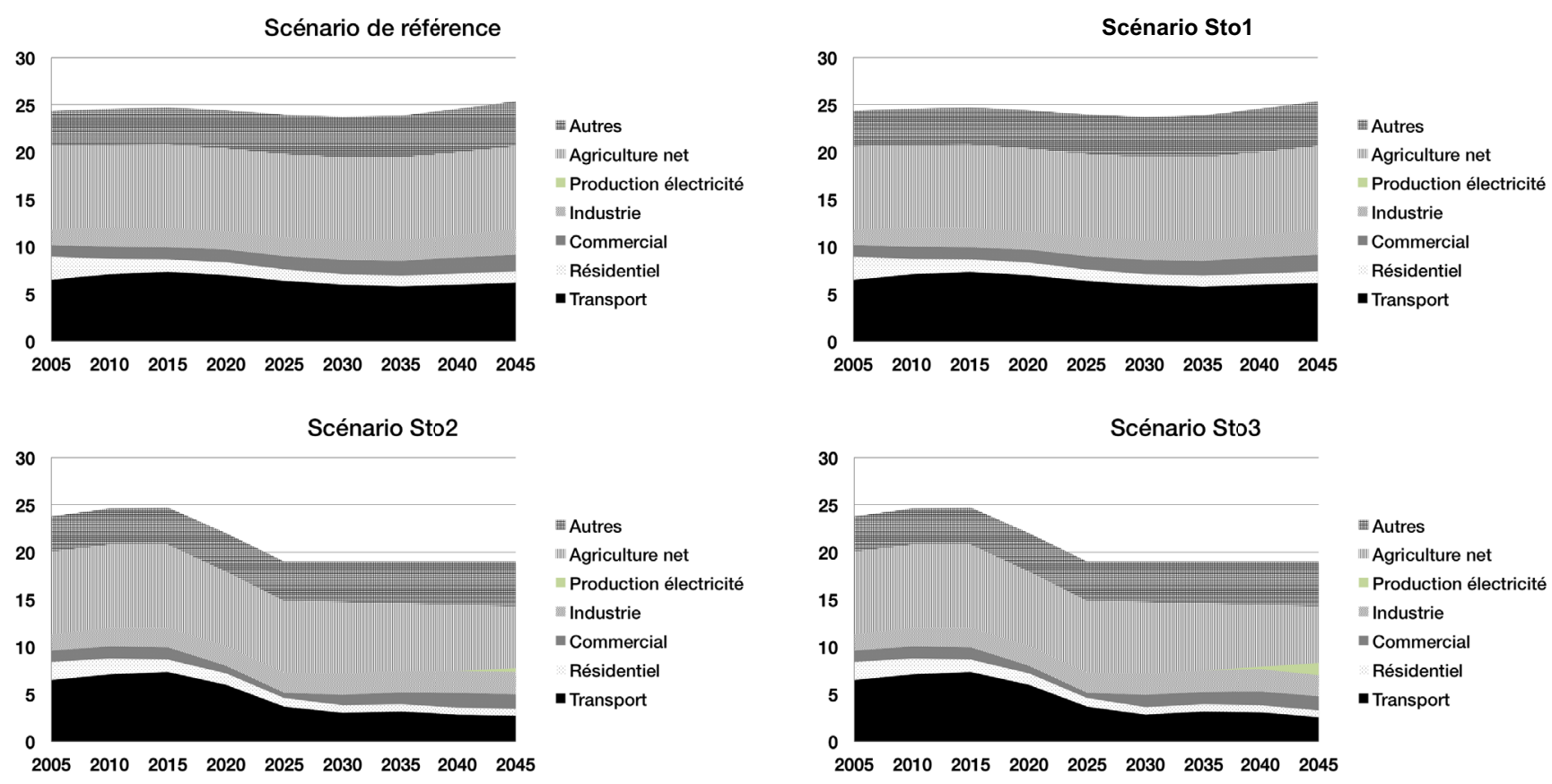

Fig. 8. Émissions de gaz à effet de serre en $\mathrm{Mt} \mathrm{CO}_{2}$-eq (scénario de référence et scénarios stochastiques robustes de la figure 7).

\section{Atténuation}

Le secteur du transport est le principal contributeur à la réduction des émissions (Fig. 8). Les secteurs commercial et résidentiel y participent également. Dans le cas d'un fort impact sur la demande utile de chauffage et de climatisation, les émissions dues à une production accrue d'électricité à partir du gaz sont compensées par une réduction plus importante dans le secteur des transports, grâce à une plus forte pénétration des biocarburants.

\section{Choix technologiques}

\section{- Transport}

C'est dans le secteur du transport que les choix technologiques sont les plus contrastés, comme on peut le constater sur la figure 9. Alors que dans le scénario de référence, la voiture hybride dominait le marché, dans les scénarios robustes avec contrainte sur les émissions de gaz à effet de serre, on voit apparaître un florilège de technologies «zéro émission ».

L'incertitude quant aux coûts des voitures entraîne, en effet, une diversification des technologies envisagées. La contrainte climatique favorise la pénétration des voitures électriques et de celles consommant des biocarburants. On remarque un effet induit par l'augmentation des demandes en climatisation qui a tendance à réduire la part du transport électrique au profit des biocarburants.

\section{- Production d'électricité}

L'incertitude quant à la disponibilité de l'éolien entraîne une baisse de cette activité par rapport au scénario de référence (Fig. 10). La pénétration de l'énergie solaire dans les scénarios stochastiques à partir de 2030 est motivée par la réduction imposée des émissions de gaz à effet de serre. En fin d'horizon, en 2040, des turbines à gaz apparaissent pour satisfaire la hausse de la demande d'électricité, du fait de la demande plus élevée en climatisation, tout en assurant les exportations d'électricité (utilisation de Golfech au plan national).

\section{- Chauffage et climatisation}

La solution robuste propose une diversification de technologies « vertes », par exemple le chauffage au bois, la géothermie, le solaire, le chauffage en réseau urbain, et, évidemment, l'isolation, comme on peut le constater sur la figure 11. La pénétration de ces technologies explique que ce secteur contribue à la baisse des émissions de gaz à effet de serre.

\section{Imports énergétiques}

La contrainte sur les émissions a pour effet de réduire les achats de produits pétroliers. Le système énergétique compense les risques liés à l'incertitude de la production électrique locale par des importations en électricité, cependant que Golfech continue de subvenir à une partie de la demande nationale. Comme nous avons pu le voir, on observe une utilisation accrue de biocarburants dans 

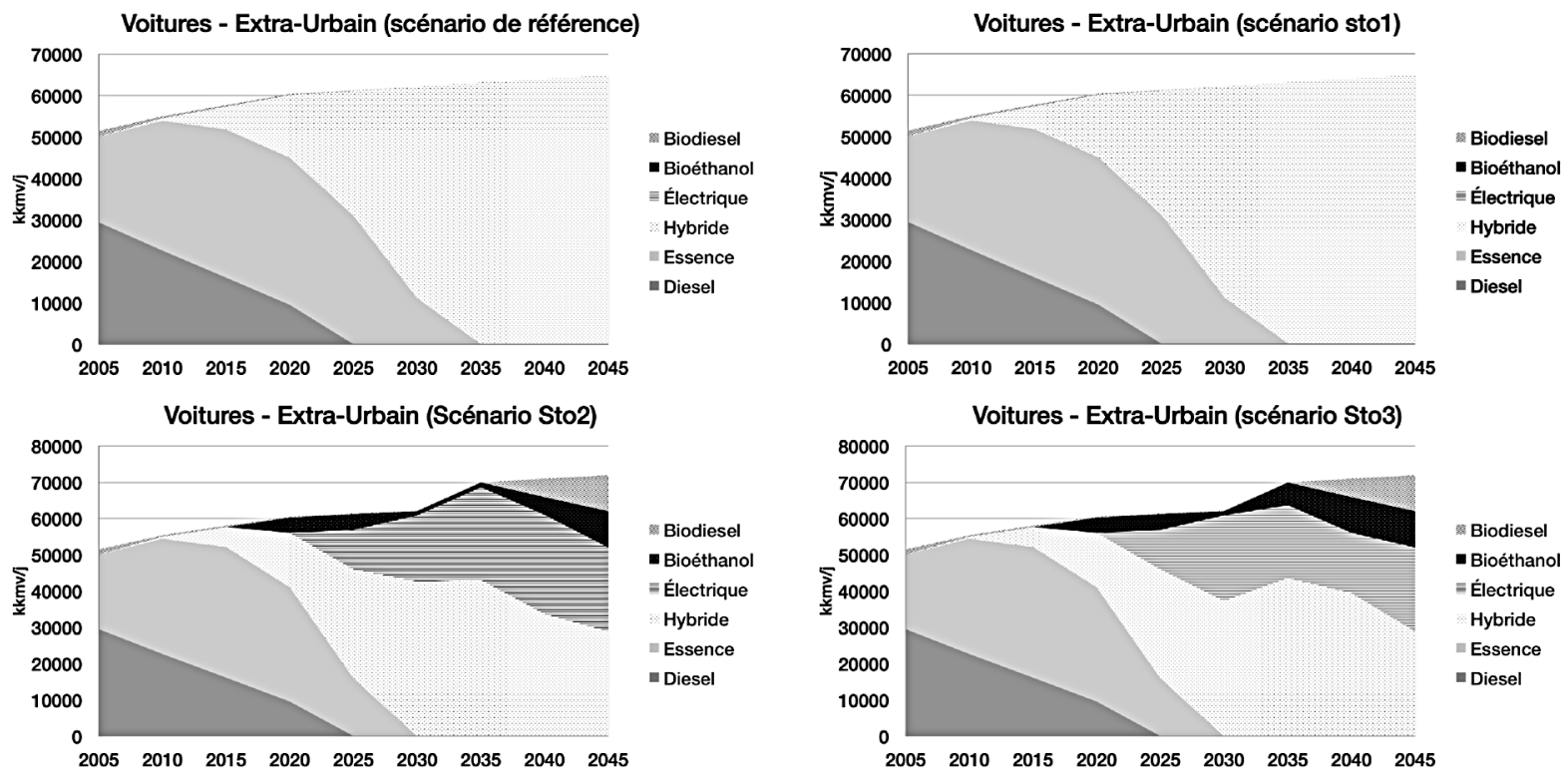

Fig. 9. Transport individuel (scénario de référence et scénarios stochastiques robustes de la figure 7).
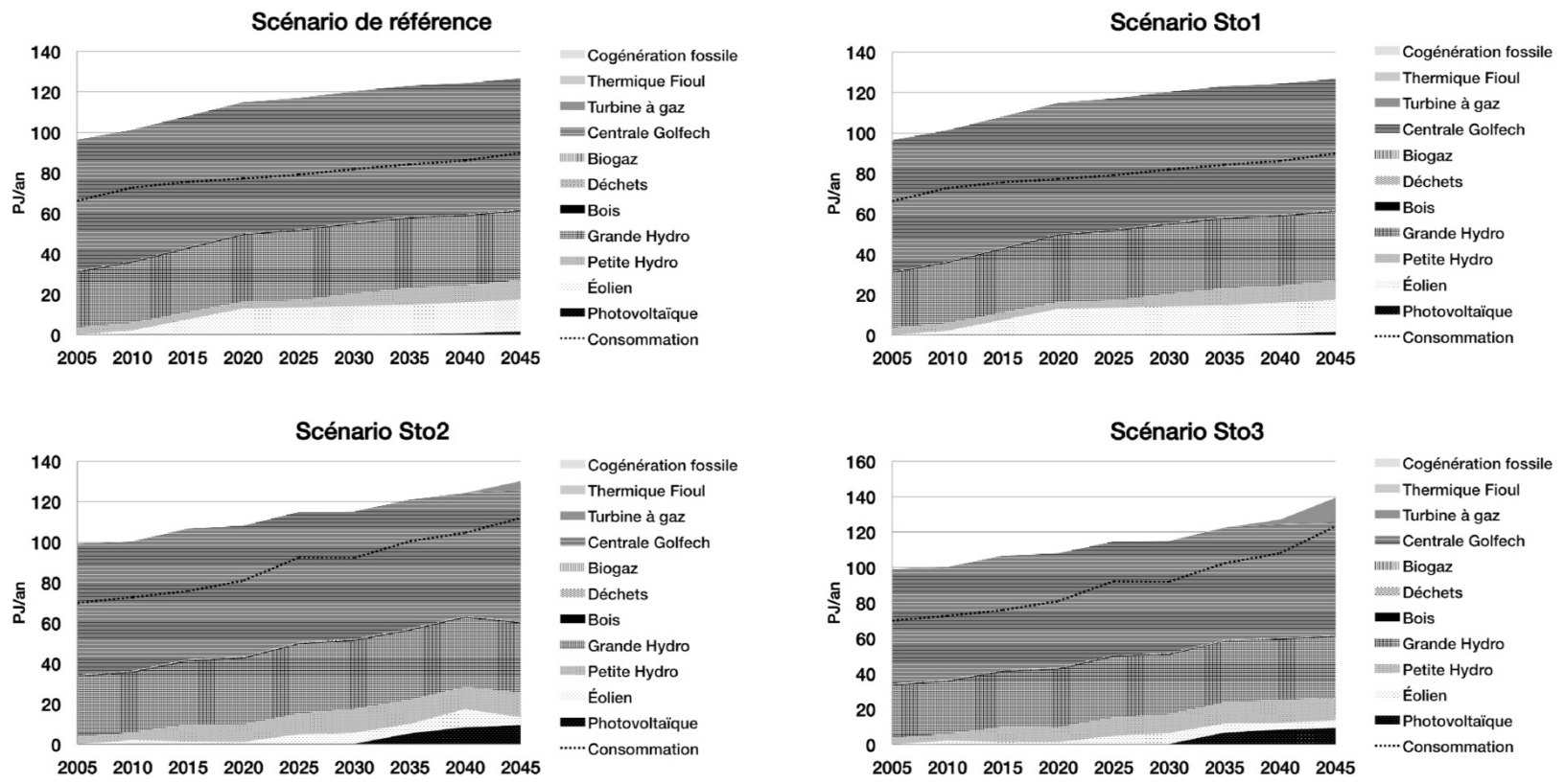

Fig. 10. Production électrique (scénario de référence et scénarios stochastiques robustes de la figure 7).

le but de libérer de l'électricité employée pour satisfaire la demande accrue en climatisation, ainsi que des achats d'hydrogène destinés au transport des camions et des bus (piles à hydrogène).

\section{Interprétation des résultats}

À l'analyse détaillée des résultats, une première remarque s'impose : les mesures d'adaptation proactive ne sont pas mises en œuvre. Le système énergétique possède des capacités d'adaptation rapide et efficace lorsque les perturbations dues au changement climatique apparaissent. En d'autres termes, le système énergétique n'a pas besoin d'anticiper les différents scénarios de demandes en climatisation et en chauffage. On observe en effet que les choix technologiques sur la période 2010-2025 dans une approche scénario par scénario coïncident avec ceux de l'approche de la programmation 

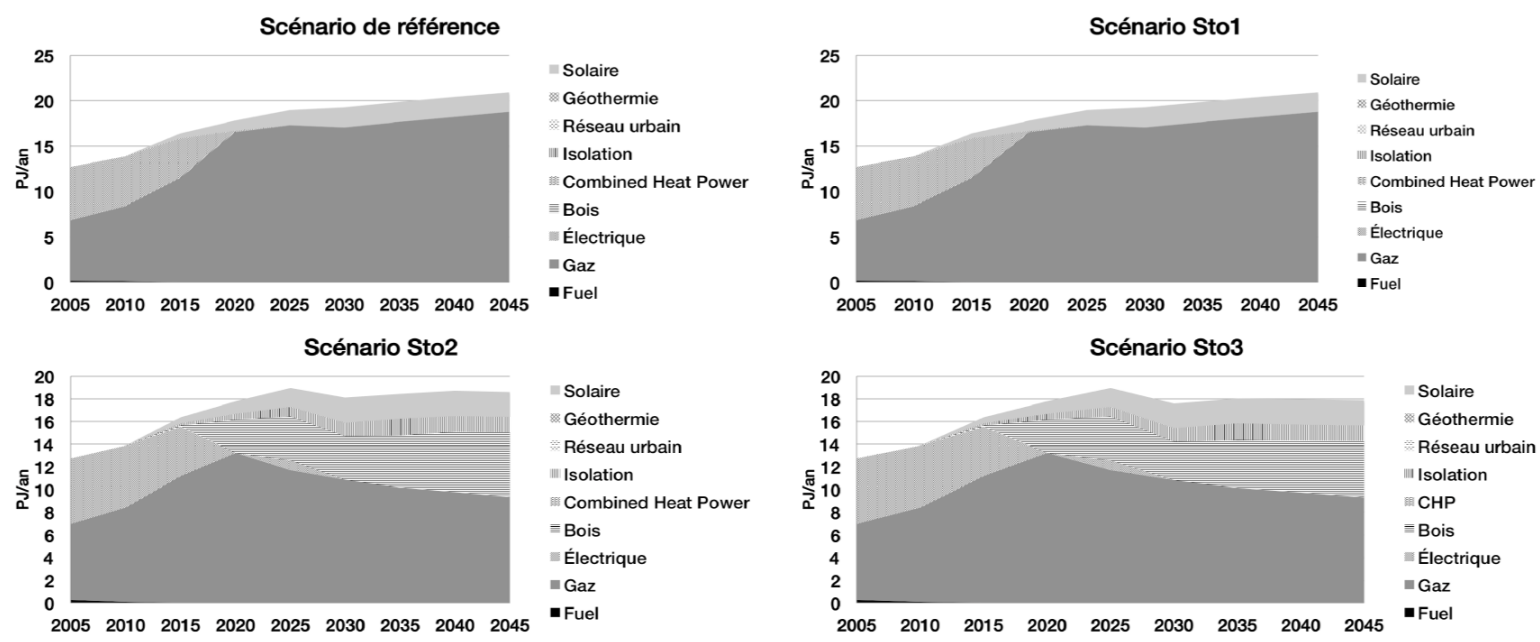

Fig. 11. Chauffage et eau chaude dans les appartements (scénario de référence et scénarios stochastiques robustes de la figure 7).

stochastique décrits ci-dessus. Les résultats étant identiques, nous ne reproduisons pas les graphes de l'approche scénario par scénario.

La combinaison de l'adaptation et de l'atténuation entraîne une hausse de la demande en électricité, compensée en partie par une progression de l'utilisation de biocarburants dans le secteur des transports ${ }^{15}$. Ainsi, les secteurs qui contribuent le plus à l'atténuation des émissions de gaz à effet de serre sont le transport individuel extraurbain ainsi que le chauffage résidentiel. À noter que l'augmentation éventuelle de la demande en climatisation se traduit également par une hausse de la demande en électricité.

La robustesse des politiques étudiées est obtenue par une plus grande diversification des choix technologiques et par conséquent du mix énergétique. C'est, par exemple, le cas dans le secteur du transport où les voitures électriques, hybrides et à biocarburant se partagent le marché.

Dans le secteur électrique, l'incertitude due à l'acceptabilité des éoliennes et à la disponibilité des petites centrales hydroélectriques se traduit par la pénétration de turbines à gaz en fin d'horizon et l'augmentation des achats d'électricité sur les marchés. Le niveau d'activité de Golfech est, par contre, maintenu, alors même que le changement climatique fait peser une incertitude quant à sa disponibilité en été. Son importance est considérée trop grande et l'on préfère jouer sur les autres sources d'incertitude pour réduire la vulnérabilité du système électrique.

\footnotetext{
15 Le modèle peut être étendu à la considération de l'adaptation du secteur agricole à une augmentation de la production d'intrants pour les biocarburants (cf. Vázquez-Rowe et al., 2013).
}

Une des principales conclusions de l'étude est que le coût de la robustesse pour le système énergétique est globalement assez réduit et ce, quel que soit le scénario climatique étudié (environ $+1,3 \%$ ). Un autre effet intéressant de la robustesse est qu'elle contribue à réduire notablement la vulnérabilité du système énergétique. Cela s'explique par le fait que les incertitudes portent sur les technologies vulnérables. Réduire l'incertitude contribue donc à réduire la vulnérabilité et vice versa. Par ailleurs, nous avons observé, dans un cas sans contrainte sur les émissions, que la robustesse a des effets négatifs sur les émissions de $\mathrm{CO}_{2}$. En effet, pour réduire l'incertitude pesant sur le système, on privilégie dans le modèle des solutions alternatives peu coûteuses et généralement émettrices telles les technologies au gaz. Dans le cas du scénario sans limite de $\mathrm{CO}_{2}$, on arrive à une augmentation de 7 à $8 \%$ des émissions en fin d'horizon. Il est donc important de combiner un objectif de réduction des émissions ainsi que robustesse et contrainte environnementale afin de diminuer la vulnérabilité du système énergétique.

\section{Conclusion}

Cette étude a fourni une démonstration des possibilités offertes par les techniques d'optimisation stochastique et robuste pour prendre en compte les activités d'adaptation et d'atténuation dans la modélisation technico-économique des systèmes énergétiques régionaux. Les activités modélisées sont essentiellement les choix technologiques destinés à satisfaire les demandes utiles et à produire l'électricité. D'autres mesures d'adaptation pourraient être prises en compte dans ce type de modèle. Par exemple, des investissements préventifs dans l'environnement bâti, dans le but de rendre les logements mieux adaptés à des vagues de chaleur, pourraient 
être modélisés et mis en concurrence avec les activités d'adaptation réactive que sont les installations de climatisation ne servant qu'une partie de l'année.

Enfin, pour des régions exposées à des événements extrêmes, tels que crues centennales, élévation du niveau de la mer ou raz de marée, les investissements en équipements de protection, digues et génératrices de secours, par exemple, pourraient être modélisés. Pour les régions possédant des centrales nucléaires à proximité de la mer, cette modélisation pourrait s'avérer riche d'enseignements.

En conclusion, cette étude montre que pour pallier l'incertitude qui entoure les besoins d'adaptation au changement climatique, la diversification des choix énergétiques et technologiques est la bonne solution économique. Le modèle ETEM-AR peut se révéler très utile pour constituer ainsi un portefeuille optimal d'options technologiques dans le cadre de la construction de plans climat territoriaux.

\section{Annexe mathématique}

\section{Modèle d'optimisation déterministe}

Soit $i$ et $j$ les indices des commodités, $K$ l'indice des technologies et $t$ l'indice des périodes, la formulation mathématique simplifiée du modèle ETEM s'écrit :

$$
\begin{gathered}
\min \quad f(X, C, I, E) \\
I_{i t}+\sum_{k} X_{i k t}^{\text {out }}=E_{i t}+\sum_{k} X_{i k t}^{i n}+d_{i t}, \quad \forall i \forall t \\
\sum_{j} \beta_{i j k t} X_{j k t}^{i n}=X_{i k t}^{\text {out }}, \quad \forall i \forall k \forall t \\
\sum_{i} X_{i k t}^{\text {out }} \leq \alpha_{k t} \gamma_{k t}\left(c_{k t}+\sum_{l \leq t} C_{k l}\right), \quad \forall k, \forall t \\
g_{m}(X, C, I, E) \leq 0, \quad \forall m
\end{gathered}
$$

avec $X=\left(X^{\text {in }}, X^{\text {out }}\right)$, les variables représentant les flux de commodités entrant et sortant des technologies, $C$ les variables d'investissement dans les capacités de technologies et $I$ et $E$ les variables d'import et d'export. La fonction objectif $f(X, C, I, E)$ représente l'ensemble des coûts et des profits annualisés fixes et variables associés aux technologies et à leur utilisation, aux investissements, aux imports et aux exports. Les contraintes (1b) garantissent la satisfaction des demandes et la conservation des flux, les contraintes (1c) lient les inputs aux outputs des technologies, et les contraintes (1c) sont des contraintes de capacité sur l'utilisation des technologies. Enfin, les contraintes $g_{m}(X, C, I, E)$ représentent l'ensemble des contraintes de bornes sur l'activité des technologies, les investissements, les capacités, les exports et les imports. Les principaux paramètres du modèle sont les suivants :

- $\alpha$ : facteur de disponibilité des technologies ;

- $\beta$ : facteur d'efficacité des technologies reliant les inputs aux outputs ;

- $\gamma$ : facteur de conversion de la capacité en énergie ;

- $d$ : vecteur de demandes.

\section{Modèle d'optimisation stochastique}

Nous donnons ici la formulation stochastique du modèle ETEM pour laquelle les scénarios indexés par $\omega$ ont un tronc commun avant la période $\bar{t}$ et se séparent ensuite avec des demandes $d_{\omega}$ différentes. À chaque scénario $\omega$, on associe une probabilité $\pi_{\omega}$ de réalisation. Ainsi, dans le modèle stochastique, on cherche à minimiser l'espérance des coûts sur l'ensemble des scénarios comme suit :

$$
\min \sum_{\omega} \pi_{\omega} f\left(X^{\omega}, C^{\omega}, I^{\omega}, E^{\omega}\right)
$$

$$
\begin{aligned}
& I_{i t}^{\omega}+\sum_{k}\left(X_{i k t}^{\text {out }}\right)^{\omega}=E_{i t}^{\omega}+\sum_{k}\left(X_{i k t}^{i n}\right)^{\omega}+d_{i t}^{\omega}, \forall i \forall t \forall \omega \\
& \sum_{j} \beta_{i j k t}\left(X_{j k t}^{i n}\right)^{\omega}=\left(X_{j k t}^{o u t}\right)^{\omega}, \quad \forall i \quad \forall k \forall t \quad \forall \omega \\
& \sum_{i}\left(X_{i k t}^{\text {out }}\right)^{\omega} \leq \alpha_{k t} \gamma_{k t}\left(c_{k t}+\sum_{l \leq t} C_{k l}^{\omega}\right), \quad \forall t \forall k \forall \omega \\
& g_{m}\left(X^{\omega}, C^{\omega}, I^{\omega}, E^{\omega}\right)(=\leq \text { ou } \geq) 0, \quad \forall m \forall \omega \\
& X_{i k t}^{\omega}=X_{i k t}^{\bar{\omega}} \text { et } C_{i k t}^{\omega}=C_{i k t}^{\bar{\omega}}, \forall i \forall k \forall \omega \forall \bar{\omega} \neq \omega \forall t \leq \bar{t} \\
& I_{i t}^{\omega}=I_{i t}^{\bar{\omega}} \text { et } E_{i t}^{\omega}=E_{i t}^{\bar{\omega}}, \forall i \forall \omega \quad \forall \bar{\omega} \neq \omega \forall t \leq \bar{t}
\end{aligned}
$$

Dans cette formulation, les variables sont indexées sur chaque scénario $\omega$ et les contraintes additionnelles $(2 \mathrm{f})$ et $(2 \mathrm{~g})$ sont appelées contraintes de non-anticipativité. Elles garantissent que les décisions prises sur chacun des scénarios seront identiques avant la période $\bar{t}$.

\section{Modèle d'optimisation robuste}

Dans cette partie, nous présentons la mise en œuvre de l'optimisation robuste aux contraintes incertaines du modèle ETEM. Nous supposons maintenant les facteurs de disponibilité des technologies $\alpha$ comme incertains. Ces facteurs définis dans l'intervalle [0,1] apparaissent dans ETEM dans les contraintes de capacité (1d).

L'approche proposée ne consiste pas à robustifier chaque contrainte de capacité (une par technologie 
incertaine) individuellement. Elle considère au contraire l'ensemble des technologies incertaines simultanément dans la définition des stratégies robustes. L'objectif est donc de permettre au système énergétique de satisfaire les demandes futures de manière globale. Afin d'implémenter cette approche, nous avons donc créé et introduit dans le modèle ETEM, pour chaque période de temps, une nouvelle contrainte qui est la somme des contraintes individuelles incertaines.

$$
\sum_{i k} X_{i k t}^{o u t} \leq \sum_{k} \alpha_{k t} \gamma_{k t}\left(c_{k t}+\sum_{l \leq t} C_{k l}\right), \quad \forall t
$$

Cette nouvelle contrainte concerne donc la capacité totale dans le système énergétique pour les technologies dites incertaines. Nous proposons par la suite de robustifier ces nouvelles contraintes. Avant d'appliquer les techniques de l'optimisation robuste, il nous faut définir un modèle d'incertitude pour les facteurs de disponibilité. Nous posons :

$$
\alpha_{k t}=\bar{\alpha}_{i t}+\xi_{k t} \tilde{\alpha}_{k t}
$$

où, pour chaque technologie $k, \bar{\alpha}_{k t}$ est la valeur nominale du facteur de disponibilité, $\tilde{\alpha}_{k t}$ sa variabilité, et $\xi_{k t}$ une variable aléatoire indépendante dans l'intervalle $[-1,1]$. Ainsi, $\left[\bar{\alpha}_{k t}-\tilde{\alpha}_{k t}, \bar{\alpha}_{k t}+\tilde{\alpha}_{k t}\right]$ donne l'intervalle de variation pour le facteur de disponibilité. Pour un $t$ donné, la contrainte (3) s'écrit alors :

$$
\sum_{i k} X_{i k t}^{o u t} \leq \sum_{k} \gamma_{k t}\left(\bar{\alpha}_{k t}+\xi_{k t} \tilde{\alpha}_{k t}\right)\left(c_{k t}+\sum_{l \leq t} C_{k l}\right)
$$

Cela est équivalent à :

$$
\begin{aligned}
\left\{\sum_{i k} X_{i k t}^{o u t}-\gamma_{k t} \bar{\alpha}_{k t}\left(c_{k t}+\sum_{l \leq t} C_{k l}\right)\right\}- \\
\left\{\sum_{k} \gamma_{k t} \xi_{k t} \tilde{\alpha}_{k t}\left(c_{k t}+\sum_{l \leq t} C_{k l}\right)\right\} \leq 0
\end{aligned}
$$

La première somme dans la contrainte (4) est une expression linéaire déterministe, tandis que la seconde est aléatoire. L'approche robuste dans Ben-Tal et al. (2009) consiste alors à remplacer la partie aléatoire de la contrainte (4) par un facteur de sécurité. L'objectif de ce facteur est de garantir la satisfaction de la contrainte pour un ensemble « raisonnable» de réalisations de l'incertitude, nommé ensemble d'incertitude. Dans ce papier, nous considérons l'ensemble d'incertitude $\Xi_{t}$, très souvent utilisé, défini par l'intersection des boules $l_{1}$ et $l_{\infty}$ :

$$
\Xi_{t}=\left\{\xi\left|\sum_{k}\right| \xi_{k t}|\leq \kappa,| \xi_{k t} \mid \leq 1, \forall k\right\} .
$$

Cet ensemble permet d'exclure du domaine les réalisations incertaines extrêmes peu pertinentes. Une solution est alors dite « robuste » si elle satisfait la contrainte robuste suivante :

$$
\begin{aligned}
& \left\{\sum_{i k} X_{i k t}^{o u t}-\gamma_{k t} \bar{\alpha}_{k t}\left(c_{k t}+\sum_{l \leq t} C_{k l}\right)\right\}- \\
& \left\{\sum_{k} \gamma_{k t} \xi_{k t} \tilde{\alpha}_{k t}\left(c_{k t}+\sum_{l \leq t} C_{k l}\right)\right\} \leq 0, \quad \xi \in \Xi_{t}
\end{aligned}
$$

L'optimisation robuste s'appuie essentiellement sur la théorie de la dualité en optimisation convexe, pour substituer la partie incertaine de la contrainte originale par son pire cas dans l'ensemble d'incertitude. La contrainte robuste est ainsi transformée en un nombre fini d'inégalités, appelé équivalent robuste. L'équivalent robuste de (5) s'écrit :

$$
\begin{gathered}
\left\{\sum_{i k} X_{i k t}^{o u t}-\gamma_{k t} \bar{\alpha}_{k t}\left(c_{k t}+\sum_{l \leq t} C_{k l}\right)\right\}+\left\{v_{t}+\kappa \sum_{k} u_{k t}\right\} \leq 0 \\
v_{t}+u_{k t} \geq \gamma_{k t} \tilde{\alpha}_{k t}\left(c_{k t}+\sum_{l \leq t} C_{k l}\right) \\
v_{t}+u_{k t} \geq-\gamma_{k t} \tilde{\alpha}_{k t}\left(c_{k t}+\sum_{l \leq t} C_{k l}\right) \\
v \geq 0 \quad u \geq 0
\end{gathered}
$$

L'équivalent robuste contient $(n+1)$ variables additionnelles (variables $u$ et $v$ ) et $2 n$ contraintes linéaires additionnelles, où $n$ représente le nombre de paramètres incertains dans la contrainte.

La mise en œuvre des techniques de l'optimisation robuste aux paramètres de coûts incertains est identique, à une exception près. L'ensemble des paramètres incertains apparaissant dans la même fonction, il n'est pas nécessaire de créer de nouvelle contrainte comme c'est le cas pour les facteurs de disponibilité.

\section{Remerciements}

Nous remercions l'Agence de l'environnement et de la maîtrise de l'énergie (Ademe) qui a financé cette étude dans le cadre du programme "Gestion et impacts du changement climatique » (GICC) du ministère de l'Environnement et du Développement durable (2012, réf. 0910 C0093) ainsi que le Qatar National Research Fund (2014-2016, réf. 6-1035-5-126). Nous tenons tout particulièrement à remercier Guillaume Tarel et Julien Thénie pour la collecte et le traitement des données concernant la région Midi-Pyrénées, Damien Pilloud pour sa contribution aux analyses climatiques et Jean-Philippe Vial pour les discussions sur le sujet de l'optimisation robuste. 


\section{Références}

Babonneau, F., Kanudia, A., Labriet, M., Loulou, R. Vial, J.-P., 2012. Energy security: a robust optimization approach to design a robust European energy supply via TIAM, Environmental Modeling and Assessment, 17, 1, 19-37.

Ben-Tal, A., El Ghaoui, L., Nemirovski, A., 2009. Robust optimization, Princeton, Princeton University Press.

Dessai, S., Hulme, M., 2004. Does climate adaptation policy need probabilities?, Climate Policy, 4, 2, 107-128.

Dessai, S., Hulme, M., 2007. Assessing the robustness of adaptation decisions to climate change uncertainties: a case study on water resources management in the east of England, Global Environmental Change, 17, 1, 59-72.

Dessai, S., Hulme, M., Lempert, R., Pielke, R., 2009. Climate prediction: a limit to adaptation, in Adger, W.N., Lorenzoni, I., O'Brien, K.L. (Eds), Adapting to climate change: thresholds, values, governance, Cambridge (UK)/New York, Cambridge University Press.

Finon, D., 1979. Scope and limitations of formalized optimisation of a national energy system, the EFOM model, in Strub, A.S. (Ed.), Energy models for the European community. An energy policy special, Guildford, IPC Science and technology Press Limited.

Fishbone, L.G., Abilock, H., 1981. Markal, a linearprogramming model for energy systems analysis: Technical description of the BNL version, International Journal of Energy Research, 5, 4, 353-375.

Fragnière, E., Haurie, A., 1996. A stochastic programming model for energy/environment choices under uncertainty, International Journal of Environment and Pollution, 6, 4-6, 587-603.

Haurie, A., Kübler, J.J.E., Clappier, A., van den Bergh, H., 2004. A metamodeling approach for integrated assessment of air quality policies, Environmental Modeling $\mathcal{E}$ Assessment, 9, 1, $1-12$.

Hawkins, E., Sutton, R., 2009. The potential to narrow uncertainty in regional climate predictions, Bulletin of the American Meteorological Society, 90, 8, 1095-1107.

Hill, D. (Ed.), 1996. The baked apple? Metropolitan New York in the greenhouse, Annals of The New York Academy of Sciences, 790.
Linnerud, K., Mideksa, T.K., Eskeland, G., 2011. The impact of climate change on nuclear power supply, The Energy Journal, 32, 1, 149-168.

Loulou, R., Kanudia, A., 1999. Minimax regret strategies for greenhouse gas abatement: methodology and application, Operations Research Letters, 25, 219-230.

Loulou, R. Labriet, M., 2008. ETSAP-TIAM: the TIMES integrated assessment model, Part I: Model structure, Computational Management Science, 5, 1, 7-40.

Loulou, R., Labriet, M., Kanudia, A., 2009. Deterministic and stochastic analysis of alternative climate targets under differentiated cooperation regimes, Energy Economics, 31, supp. 2, 131-143.

Oremip (Observatoire régional de l'énergie de Midi-Pyrénées), 2013. Chiffres clés de l'énergie et des gaz à effet de serre en MidiPyrénées 2011, Oremip.

Région Midi-Pyrénées, 2011. Schéma régional climat-air-énergie (SRCAE) de Midi-Pyrénées: projet de schéma soumis à consultation. Document principal et annexes, Région MidiPyrénées.

Thénie, J., van Delft, C., Vial, J.-P., 2007. Automatic formulation of stochastic programs via an algebraic modeling language, Computational Management Science, 4, 1, 17-40.

Vázquez-Rowe, I., Rege, S., Marvuglia, A., Thénie, J., Haurie, A., Benetto, E., 2013. Application of three independent consequential LCA approaches to the agricultural sector in Luxembourg, The International Journal of Life Cycle Assessment, 18, 8, 1593-1604.

Wene, C.-O., 1980. The optimum mix of conservation and substitution: an example from retrofitting of old buildings, International Journal of Energy Research, 4, 3, 271-282.

Wene, C.-O., Andersson, O., 1983. Long-range community energy planning: case study Jönköping (in Swedish), Efn/AES, Stockholm, Energiforskningsnämnden.

Wene, C.-O., Rydén, B., 1988. A comprehensive energy model in the municipal energy planning process, European Journal of Operational Research, 33, 2, 212-222. 